\title{
Dedifferentiated Liposarcoma - A Case Report
}

\author{
Shaista Choudhary ${ }^{1}$, H T Jayaprakash ${ }^{2}$, B S Ramesh ${ }^{3}$, Sida Tagore ${ }^{4}$ \\ Samiksha Ray ${ }^{5}$ \\ 1. Associate professor, dept of Pathology \\ 2. Professor \& h.o.d, dept of Pathology \\ 3. Professor dept of Surgery \\ 4. Junior resident pathology \\ 5. Senior resident pathology.
}

\begin{abstract}
Lipasarcomas are mesenchymal tumors and most common type of sarcomas occuring in adults.Dedifferentiation can occur in liposarcoma as a progression of disease or in recurrent cases. We present a case of dedifferentiated liposarcoma of thigh where dedifferentiation was seen in primary tumor itself which suggested that it was a long duration tumor.The varied histopathological findings are the hallmark of this case.
\end{abstract}

Keywords: dedifferentiated liposarcoma, malignant tumor, thigh mass.

\section{Introduction:}

Lipomatous tumors are the most common soft tissue tumors ${ }^{[1]}$.Liposarcoma is the most common soft tisssue sarcoma in adults.About $24 \%$ occur from limbs and $45 \%$ originate from retroperitoneal area.Among mesenchymal tumors liposarcoma are probably unsurpased by their wide range in morphology and behaviour ${ }^{[2]}$. They are subclassified into five histological types:Well differentiated liposarcoma(WDLS),Dedifferentiated liposarcoma(DDLS),Pleomorphic,Myxoid and Round cell liposarcoma.This classification based on histopathological findings is an important prognosticator in liposarcomas. We present a case of huge mass in thigh that was subsequently reported as DDLS.

Case Presentation: A 75 years old male patient was admitted with a huge mass in left thigh.On examination there was tenderness and reduced mobility of the affected side.Routine investigations were done which were in normal limits except for anaemia(Hb-10.5gms\%).Ultrasound showed presence of a soft tissue tumor.Patient underwent wide excision surgery.Grossly specimen consisted of two irregular grey white to grey yellow masses each one measuring $14 \times 10 \times 5 \mathrm{cms}$. Cut surface of both masses showed similar appearence consisting of fibrofatty ,cartilagenous ,myxoid and fish flesh like areas(figure1). Microscopy showed lobulated areas consisting of mature fat cells with univacuolated lipoblasts.Intervening stroma showed myxoid tissue with thin elongated cells arranged in vague fascicular pattern at focal areas.Cells in myxoid area showed nuclear hyperchromatism and pleomorphism with few giant cells(figure3).

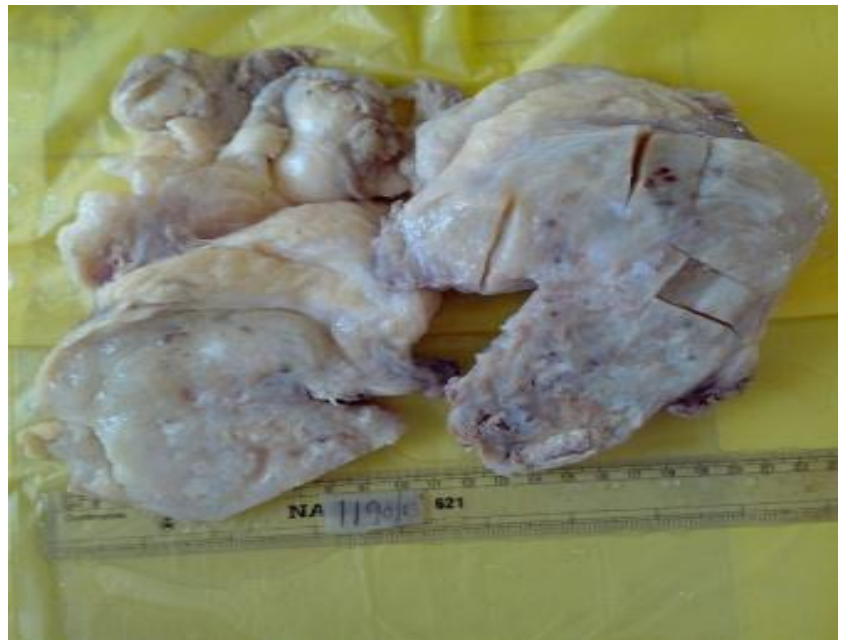

FIGURE 1: Gross appearence of excised mass showing myxoid,fibrofatty, cartilagenous and fish flesh like areas. 


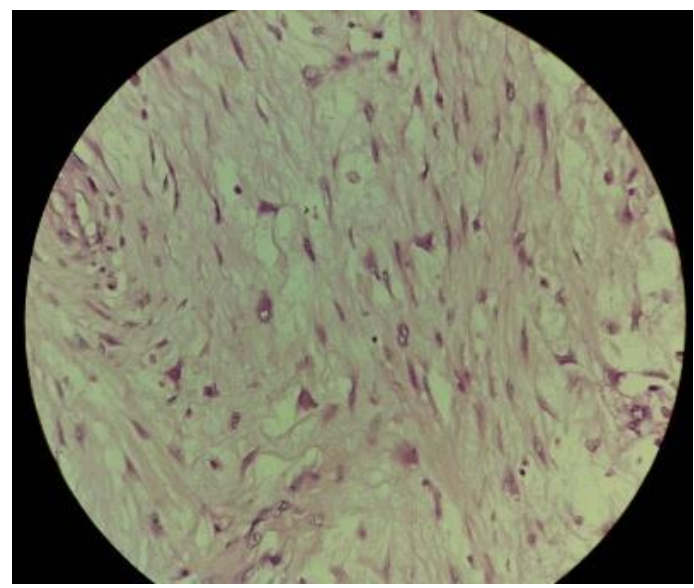

FIGURE 2: shows pleomorphism in fibroblast and also rhabdomyomatous differentiation

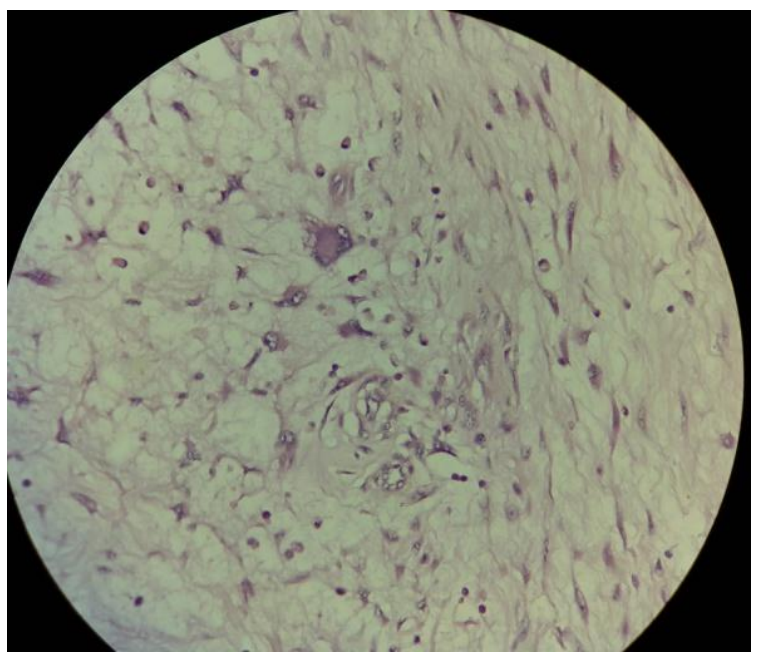

FIGURE3:showing giant cells in myxoid stroma.

Focal area in myxoid tissue showed divergent differentiation towards rhabdomyomatous and chondroid elements(figure2 \&3). The myxoid tissue was hypocellular and showed features of malignant fibrous histiocytoma.Lipomatous area showed a gradual transition to dedifferentiated area.Impression of dedifferentiated liposarcoma was made(figure4).

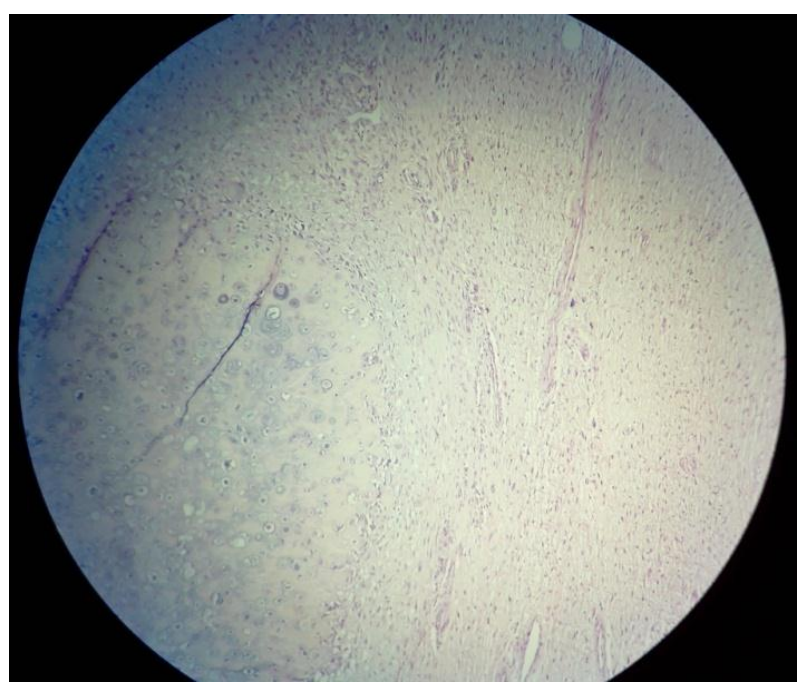

FIGURE 4: showing focal cartilagenous differentiation 


\section{DISCUSSION:}

Dedifferentiation occurs in upto $10 \%$ of well differentiated liposarcomas with predilection for retroperitoneum. ${ }^{[3]}$ The phenomenon of dedifferentiation or progression to a higher grade and less well differentiated neoplasm was first described by $\operatorname{Dahlin}^{[2]}$.This terminology was further described in detail by Evans $^{[4]}$.This case showed focal area of rhabdomyomatous differentiation.The finding of heterologous rhabdomyoblastic elements has been described in very few cases ${ }^{[4]}$. Though as earlier mentioned that dedifferentiated liposarcomas are usually seen in retroperitoneum but there is documentation of other sites like extremities too ${ }^{[5]}$.Tumour location, size and histologic subtype are the most important prognostic indicators.Dedifferentiated liposarcomas often gives rise to widespread metastasis ${ }^{[5]}$.A study was done on 155 cases of DDLS by Henricks WH et.al, to define the clinicopathologic features and behaviour of these tumors. The authors concluded that low grade dedifferentiation represents a precursor lesion of high grade differentiation ${ }^{[6]}$. This suggests that one must do extensive sampling in such cases.It would affect the prognosis.DDLS have also been reported in gastrointestinal tract i.e colon ${ }^{[7]}$, as paratesticular mass ${ }^{[8]}$ and also in liver ${ }^{[9]}$.Liposarcomas have five histological subtypes :well differentiated ,dedifferentiated,pleomorphic,myxoid and round cell type.Amongst all subtypes DDLS carries worst prognosis.Dedifferentiation signifies high grade lesion and it occurs in metastatic foci though primary DDLS have also been documented.

The present case was a primary tumor since patient did not have any previous treatment or surgery for the same lesion.It occured as a slowly growing mass which lead to restricted mobility.Thus it was thought to be a denovo phenomenon also known as primary dedifferentiation ${ }^{[10]}$.At molecular level,retinoblastoma protein has a major role to play in dedifferentiation and "two hit mechanism" is involved in the altered retinoblastoma protein expression in dedifferentiated liposarcoma ${ }^{[8]}$.According to WHO(World health organization)low grade DDLS is defined as bland spindle cells with fascicular pattern and cellularity intermediate between well differentiated sclerosing liposarcoma and usual high grade areas. The behaviour of dedifferentiated liposarcoma is that of a high grade sarcoma ${ }^{[6]}$.In conclusion we would like to mention that dedifferented liposarcoma is an aggressive tumor irrespective of its grade of dedifferentiation.Dedifferentiation in liposarcomas is a course towards progression of lesion and also signifies the long standing or even reccurent nature of disease.Hence identification of dedifferentiated components is important.

\section{References:}

[1]. Costea R, Vasiliu E, Zamescu NO, Hasouna M and Neagu S. Large thigh liposarcoma-Diagnostic and therapeutic features.J Med Life. 2011;4(2):184-188.

[2]. Enzinger and Weiss's.Liposarcoma, in: Soft Tissue Tumors, IV ed (Springer2001) 641-643.

[3]. Hoshi M, Matsumoto S, Manabe Jun, Tanizava T, Shigemitsu T, Koyanagi H .et.al.Surgery for dedifferentiated liposarcoma,presenting two radiographically and pathologically distinctive patterns.Japanese $J$ of clinical Oncology 2006;36(7):462-467.

[4]. Shanks J H, Bannerjee S S and Eyden B P.Focal rhabdomyosarcomatous differentiation in primary liposarcoma.Jour of Clin Pathol 1996; 49:770-772.

[5]. Rosai and Ackermann.Soft Tissues,in:Rosai and Ackermann's surgical pathology $9^{\text {th }}$ edition (Missouri ;Elsievior 2004$) 2283-2284$.

[6]. Henricks WH, Chu Y C, Goldblum J R.Weiss S W.Dedifferentiated Liposarcoma: a clinicopathological analysis of 155 cases with a proposal for an expanded definition of dedifferentiation.American J of Surg Pathol 1997; 3:271-281.

[7]. Turkoglu M A, Elpek G O,Dogru V,Calus H, Ucar A, Arici Cumhur. An unusual case of primary colonic dedifferentiated liposarcoma.International jour of surg case reports.2013

[8]. Ghosh A,Swami R, Sen P K, Dwaka S.Unusual presentation of dedifferentiated liposarcoma as paratesticular mass. Indian Jour of Pathol \& Microbiology2008;51(1):42-44.

[9]. Yong il kim,Eun Sil Yu,keun Wook Lee, Eui U Park,Hyung Geun Song.Dedifferentiated liposarcoma of the liver.Cancer1987;60:2785-2790.

[10]. Rugvedita S Parakh,Meera P Zawar,Pradeep A Gadgil,Neeraja S Kaujalagi.Dedifferentiated liposarcoma of the retroperitoneum:histologically low grade type.Indian Jour of Pathol \& Microbiology2010;53(2):353-355.

[11]. Samuel singer,Nicholas D.Socci,Grazia Ambrosini,Elliot Sambol,Penelope Decoralis, Yuhsin Wu .et.al.Gene expression profiling of liposarcoma identifies distinct biological types/subtypes and potential therapeutic targets in well-differentiated liposarcoma.Cancer Research2007;67:6626.

[12]. Bharat Rekhi, Pooja Navale, N.A.Jhambekar.Critical histopathological analysis of 25 dedifferentiated liposarcomas,including uncommon variants,reviewed at a tertiary refferal centre.Indian Jour of Pathol \& Microbiology2012;55(3):301. 